\title{
Second breast cancers in a Tuscan case series: characteristics, prognosis, and predictors of survival
}

\author{
S Ciatto ${ }^{1,4}$, N Houssami ${ }^{* 1,2,4}$, F Martinelli', R Bonardi', FH Cafferty ${ }^{3}$ and SW Duffy ${ }^{3}$ \\ 'Centro per lo Studio e la Prevenzione Oncologica (CSPO), Istituto Scientifico della Regione Toscana, Florence, Italy; ${ }^{2}$ Screening and Test Evaluation \\ Program (STEP), School of Public Health, University of Sydney, Sydney, Australia; ${ }^{3}$ Department of Mathematics and Statistics, Centre for Epidemiology, \\ Cancer Research UK, Wolfson Institute of Preventive Medicine, London, UK
}

Little is known about long-term outcomes following a second breast cancer diagnosis. We describe the epidemiology, characteristics and prognosis of second breast cancers in an Italian cohort. We identified women with two breast cancer diagnoses from 24278 histology records at a Tuscan breast cancer service between 1980 and 2005, and determined their survival status. Disease-specific survival from second diagnosis was examined using Cox regression analyses. Second cancers were identified in 1044 women with a median age of 60 years. In all 455 were ipsilateral relapses and 589 were contralateral cancers. Median time between first and second diagnosis was 63.4 months. The majority of second cancers was small invasive or in situ tumours. Estimated 10 -year survival from a second cancer diagnosis was $78 \%$. Survival was poorest when the second cancer was large $(H R=2.26)$ or node-positive $(H R=3.43)$, when the time between the two diagnoses was $<5$ years $(H R=1.45)$, or when the diagnosis was in an earlier epoch $(H R=2.20)$. Second tumours were more likely to be large or node-positive if the first breast cancer had these features. Prognosis following a second breast cancer in this cohort was generally good. However, large or node-positive second tumours, and shorter intervals between diagnoses were indicators of poorer survival.

British Journal of Cancer (2008) 99, 539-544. doi:I0.1038/sj.bjc.660448 I www.bjcancer.com

Published online 15 July 2008

(c) 2008 Cancer Research UK

Keywords: breast cancer; contralateral cancer; disease-specific survival; ipsilateral breast relapse; prognosis

Breast cancer $(\mathrm{BC})$ is estimated to be the most prevalent cancer in the world (Parkin et al, 2005). Earlier diagnosis and better therapy have improved survival from BC. Information relevant to the care of this group of such women, who have an elevated risk of a second $\mathrm{BC}$, is relevant to population health and clinical practice. Few studies have examined a second BC in well-defined series, and little is known about the characteristics and long term outcome in affected women (Grunfeld et al, 2002).

We report on the largest clinically defined cohort (to date) of second BC events, based on consecutive cases in a major breast service in Tuscany, and including women with ipsilateral breast relapse (IBR) and contralateral breast cancer (CBC). We aim to describe the epidemiology and clinical characteristics of second BCs, and to identify factors associated with poorer survival following a second $\mathrm{BC}$ diagnosis. The focus of our study is therefore prognosis following second $\mathrm{BC}$ events and not predictors of its occurrence.

*Correspondence: Dr N Houssami, Screening and Test Evaluation Program (STEP), School of Public Health, Edward Ford Building (A27), University of Sydney, NSW 2006, Australia or Dr S Ciatto Centro per lo Studio e la Prevenzione Oncologica (CSPO), Istituto Scientifico della Regione Toscana, Florence, Italy;

E-mails: nehmath@med.usyd.edu.au or s.ciatto@cspo.it

${ }^{4} \mathrm{Dr}$ Ciatto and Dr Houssami have equally contributed to the development and reporting of this work

Received 14 April 2008; revised 4 June 2008; accepted 5 June 2008; published online 15 July 2008

\section{MATERIALS AND METHODS}

Eligible participants consisted of all women with a prior primary BC (invasive or in situ) who were diagnosed with a second metachronous BC ( $\geqslant 6$ months after the first cancer) in 1980-2005 at the study centre. We searched the clinical and pathology archives of the Centro per lo Studio e la Prevenzione Oncologica (CSPO), identifying women with histology records (surgical or needle histology) indicating two BC diagnoses separated by at least 6 months. Medical records were then reviewed to verify eligibility. Centro per lo Studio e la Prevenzione Oncologica is Florence's main breast screening and diagnostic service, and is the only centre in the region that provides follow-up services for women with BC. Surveillance consists of two-view mammography complemented by clinical examination (with more frequent clinical examination in the initial 5 years in women treated with breast conservation). Centro per lo Studio e la Prevenzione Oncologica archives have ongoing data linkage to population cancer and mortality registries in the Tuscan region.

We use the term second BC ('second cancer') when describing all cases, but define two subgroups eligible for inclusion: women with (1) ipsilateral breast relapse (IBR), who developed a second cancer in the breast that was previously affected (including those with breast relapse and concomitant axillary disease); and (2) women with $\mathrm{CBC}$, who developed a second cancer in the opposite breast. Women presenting with metastatic cancer at diagnosis of the second $\mathrm{BC}$, who represent a very small proportion of women, were not considered for this study as histology verification is inconsistently available in these cases; inclusion might bias 
estimates of survival from second cancer diagnosis; and owing to difficulty in ascertaining whether the metastases were related to the first or second cancer event. Women presenting primarily with nodal metastases or chest wall recurrences (following mastectomy for the first cancer) were not eligible for inclusion in this study.

Data retrieved from clinical records included date of birth; date of diagnosis, histology and pathological $\mathrm{T}$ and $\mathrm{N}$ category of the first BC; date of diagnosis, side, histology and pathological $\mathrm{T}$ and $\mathrm{N}$ category of the second cancer; presence or absence of symptoms at diagnosis of second cancer; surgical management; and date of last follow-up. Survival status was assessed directly for patients regularly followed up at CSPO or according to the regional Mortality Registry for cases lost to active follow-up. Linkage with the Mortality Registry for cause of death was complete to 31 December 2005, and ascertainment of outcomes was $97 \%$ complete to 31 December 2006. Data on treatment were not available for this study, but in our setting, it comprises radiotherapy following breast conservation and (since about 1988) adjuvant systemic therapy.

\section{Statistical analysis}

Analyses are presented for all second cancers collectively and for each group (IBR and CBC). Descriptive data are reported on key variables including histology and tumour stage distribution for both the first and the second cancers. Time between occurrence of the first cancer and histological diagnosis of a second cancer ('disease-free interval', DFI), and time from second diagnosis to BC death, were calculated. When comparing features of the second tumour with features of the first tumour, data are paired and the McNemar's $\chi^{2}$ test was used. When comparing the IBR cases with the CBC cases, groups are independent and the $\chi^{2}$ test was used.

To determine the prognostic effect of features of the second cancer, we examined disease-specific survival from the second cancer diagnosis. Survival from diagnosis of the first cancer will be the subject of a separate study. For subjects who were alive at the end of follow-up or had died from a different cause, observations were censored at date of their last observation (normally 31 December 2006) or date of death. Ten-year survival was estimated using the Kaplan-Meier method. Multivariate Cox regression analysis was performed using the following covariates: (a) age at second cancer diagnosis ( $<50,50-69, \geqslant 70$ years); (b) time from first to second cancer (DFI); (c) stage ( $\mathrm{pT}$ and $\mathrm{pN}$ category) of the second cancer; (d) time period in which the second cancer was diagnosed (1980-97, 1998-2005); and (e) type of second cancer (IBR or CBC). Models were fitted separately for IBR and CBC cases, as well as for all second cancers. Results are expressed in terms of hazard ratios (HR) and 95\% confidence intervals (CI).

The Cox regression model was used to identify features of the second tumour that had the most significant adverse influence on prognosis following its diagnosis. Multivariate logistic regression analyses were then used to identify which features of the first BC predicted such second $\mathrm{BC}$. The covariates under consideration were (a) age at first cancer diagnosis $(<50,50-69, \geqslant 70$ years); (b) stage of the first cancer (pT and pN category); and (c) histology of the first cancer (invasive ductal, DCIS, other invasive). Note that this analysis is designed to predict particular features of a second tumour and not the occurrence of a second tumour.

All statistical analyses were performed using the STATA software, release 8.0 .

\section{RESULTS}

Second BCs were identified in 1044 women from 24278 breast histology records: IBR in 455 subjects and CBC in 589 (Table 1). The median age at diagnosis of the second cancer was 60 years (IQR $51-70$ ). Follow-up was available in all but 36 cases (20 IBR,
$16 \mathrm{CBC}$ ), which were excluded from outcome analyses but retained in descriptive analyses. The median follow-up period from the occurrence of the first cancer was 13.7 (IQR 9.0-18.1) years. There were $181 \mathrm{BC}$ deaths and 62 from other causes (Table 1). Second cancers were more likely to be detected asymptomatically $(67.0 \%)$ than symptomatically $(33.0 \%, P<0.001)$. However, the proportion of symptomatic cases did not differ between IBR and $\mathrm{CBC}$ $(P=0.21$, Table 1$)$.

The majority of second cancers was small invasive or in situ (77\% were pTis or pT1). DCI comprised $12 \%$, invasive ductal $56 \%$ and other histological types $32 \%$. Of CBC cases, $56 \%$ were node negative, $20 \%$ were node positive, and in $24 \%$ node status was unknown. There was a significant difference between IBR and CBC with respect to the histological type of the tumour $(P<0.0001)$, more IBR cases being DCIS, whereas more CBC cases were invasive lobular. Similarly, there was a significant difference between the two groups in the histological types of the first tumour $(P<0.0001)$; more IBR had been diagnosed with DCIS previously compared with those with CBC (17.1 vs 5.8\%). Significant differences in the distribution of $\mathrm{pT}$ categories between IBR and $\mathrm{CBC}$ were also seen for both first and second tumours (Table 1).

Table 2 summarises data on size (pT) of second tumours according to the size of the first tumour and the type of second cancer (IBR or $\mathrm{CBC}$ ). For women diagnosed with $\mathrm{CBC}$, the second tumour tended to be smaller than the first. Of those with known tumour size of both first and second cancers, $80.5 \%(409 / 508)$ of second cancers were pT1 or smaller, compared with $58.5 \%$ $(297 / 508)$ of first cancers $(P<0.0001)$. However, this difference was not observed in women with IBR, whose second tumour was more likely to be of a similar size to the first tumour $(83.3 \%(334 / 401)$ of second cancers and $81.5 \%(327 / 401)$ of first cancers were pT1 or smaller, $P=0.5$ ).

Figure 1 shows Kaplan-Meier survival curves for IBR, CBC and all second cancers. The estimated 10-year survival rate from IBR was $77.9 \%$, from CBC, $77.3 \%$ and overall in second cancers $77.6 \%$. In univariate analysis significantly poorer survival was observed with younger ( $<50$ years) and older ( $>69$ years) age at second cancer diagnosis $(P=0.03)$, a short interval from first to second cancer $(P=0.001)$, larger tumour size $(P<0.0001)$, positive nodes $(P<0.0001)$, and cancers diagnosed during the earlier time period $(P<0.0001)$. These effects were similar for IBR and CBC cases and so both groups were included in the final multivariate model. The multivariate Cox regression model is presented in Table 3 showing the proportional hazard ratios (HR) and 95\% CI for second BC variables. The strongest predictor variables for survival in this model were $\mathrm{pT}$ and $\mathrm{pN}$ categories, although time between first and second cancer, and epoch of diagnosis of second cancer, also remained significant. There was no evidence of a difference in survival between IBR and CBC.

The Cox model indicates that second cancers that were $2 \mathrm{~cm}$ or larger or node positive had a poor prognosis. Table 4 summarises multivariate analysis of the features of the first cancer that predicted these features in the second tumour: women whose first cancer was large or node positive at diagnosis were more likely to have large or node-positive second cancer.

\section{DISCUSSION}

The characteristics of second BCs in women presenting to a major centre in Tuscany represents the largest series to date to include both IBR and $\mathrm{CBC}$, with data on outcomes in almost all subjects. As BC survival is improving, clinicians will be providing care to increasing number of women at risk of developing a second cancer in either breast. Women who experienced IBR or CBC in this study were on average around 60 years of age when they experienced the second $\mathrm{BC}$ event. This is not an old population in the context of life 


\begin{tabular}{|c|c|c|c|c|c|}
\hline Variable & Category/quantity & $\begin{array}{c}\text { IBR } \\
(N=455)\end{array}$ & $\begin{array}{c}\text { CBC } \\
(N=589)\end{array}$ & $\begin{array}{l}\text { All second } \\
\text { cancers } \\
(N=1044)\end{array}$ & $P$-value ${ }^{a}$ \\
\hline $\begin{array}{l}\text { Time from second cancer to breast } \\
\text { cancer death }\end{array}$ & Median (IQR), years & $3.1(1.9-5.3)$ & $3.8(1.9-6.2)$ & $3.3(1.9-5.7)$ & NA \\
\hline Breast cancer deaths & & 78 & 103 & 181 & NA \\
\hline Deaths from other causes & & 14 & 48 & 62 & NA \\
\hline Time from first to second cancer (DFI) & Median (IQR), months & $57.4(30.5-107.6)$ & $68.0(36.0-122.1)$ & $63.4(33.2-117.0)$ & 0.029 \\
\hline Age at second cancer & Median (IQR), years & $59(50-69)$ & $61(53-7 \mid)$ & $60(5 I-70)$ & 0.082 \\
\hline \multirow[t]{2}{*}{ Presentation of second cancer } & Asymptomatic (\%) & $314(69)$ & $385(65)$ & $699(67)$ & 0.214 \\
\hline & Symptomatic (\%) & $|4|(3 \mid)$ & $204(35)$ & $345(33)$ & \\
\hline \multirow[t]{3}{*}{ pT category of second cancer } & pTis (\%) & $70(15)$ & $55(9)$ & $125(12)$ & 0.015 \\
\hline & $\mathrm{pTIa}-\mathrm{c}(\%)$ & $283(62)$ & $397(67)$ & $680(65)$ & \\
\hline & $\mathrm{pT} 2+(\%)$ & $71(16)$ & $105(18)$ & $176(17)$ & \\
\hline \multirow[t]{5}{*}{ Histology of second cancer } & DCIS (\%) & $72(16)$ & $55(9)$ & $127(12)$ & $<0.0001$ \\
\hline & Invasive ductal (\%) & $238(52)$ & $346(59)$ & $584(56)$ & \\
\hline & Invasive lobular (\%) & $55(12)$ & $115(20)$ & $170(16)$ & \\
\hline & Other special types (invasive) ${ }^{c}(\%)$ & $22(5)$ & $43(7)$ & $65(6)$ & \\
\hline & Other breast cancers (\%) & $68(15)$ & $30(5)$ & $98(9)$ & \\
\hline \multirow[t]{3}{*}{ Surgery for second cancer } & Mastectomy (\%) & $248(55)$ & $233(40)$ & $481(46)$ & NA \\
\hline & WLE (\%) & $183(40)$ & $342(58)$ & $525(50)$ & \\
\hline & Data missing (\%) & $24(5)$ & $14(2)$ & $38(4)$ & \\
\hline \multicolumn{6}{|l|}{ Features of first cancer } \\
\hline \multirow{4}{*}{$\begin{array}{l}\text { Age at first cancer } \\
\text { PT category of first cancer }\end{array}$} & Median (IQR), years & $51(43-63)$ & $53(45-62)$ & $53(44-62)$ & 0.035 \\
\hline & pTis (\%) & $85(19)$ & $37(6)$ & $122(12)$ & $<0.000$ \\
\hline & $\mathrm{pT} \mathrm{a}-\mathrm{c}(\%)$ & $257(56)$ & $27 \mid(46)$ & $528(5 \mathrm{I})$ & \\
\hline & $\mathrm{pT} 2+(\%)$ & $85(19)$ & 227 (39) & $312(30)$ & \\
\hline
\end{tabular}

$\overline{\mathrm{DCIS}}=$ ductal carcinoma in situ; IQR = interquartile range; $\mathrm{NA}=$ not applicable. ${ }^{\mathrm{a}}$ Comparison of IBR with CBC where applicable. ${ }^{\mathrm{b}} \mathrm{Based}$ on I0I6 subjects (from I044 subjects) who had at least one episode of follow-up (from second cancer diagnosis) and verified outcomes through linkage with regional cancer or mortality registry. ${ }^{C}$ Includes cases where histological type of breast malignancy was not specified and those with missing data on type of cancer histology.

expectancy in developed countries. These cancers are more likely to be diagnosed in asymptomatic women, and are generally smaller tumours than the initial cancer in the same women.

Overall 10-year survival from diagnosis of the second cancer was $77.6 \%$, and the median time between first and second cancer diagnosis was 63.4 months. A shorter time from first till second cancer diagnosis was associated with poorer survival. Ten-year survival estimates in our study are similar to survival estimates of $75 \%$ observed for first invasive cancers in the Swedish two-county study (Tabar et al, 1992), and the $73 \%$ observed in first invasive cancers diagnosed in the 1990s in the West Midlands, UK (G Lawrence, personal communication).

Many studies have looked at IBR and reported on factors predicting local recurrence. We have taken a different approach by focusing on the identification of features associated with prognosis once a second $\mathrm{BC}$ has occurred (rather than predictors of recurrence) and have included $\mathrm{CBC}$ as well as IBR. Most studies of CBC have included small number of cases and limited follow-up (Grunfeld et al, 2002). The exception to this is a study that reported on stage-related survival in CBCs only and showed good prognosis in early-stage tumours (Schootman et al, 2006). We recognise that IBR and $\mathrm{CBC}$ are essentially different events and for this reason we have reported data separately in the two groups. Our summary of key features (Table 1) highlights both similarities and differences in second cancers. Variables associated with prognosis in survival analysis were similar for both IBR and CBC. We were unable to report on tumour grade and hormone receptor status because of incomplete or non-availability of such data. It should be noted that Rack and colleagues, in a study of locoregional breast relapse found that tumour grade was not independently associated with risk of death following relapse (Rack et al, 2003).

The distribution of times between first and second cancer diagnosis in this cohort (both IBR and $C B C$ ) provides evidence supporting long-term follow-up of women after BC. Currently, some groups recommend only limited follow-up (National Institute for Clinical Excellence, 2002; The Association of Breast Surgery@BASO, 2005). However, our study suggests that a 
Table 2 Pathological tumour size ( $\mathrm{pT}$ ) category of the second breast cancer according to size of the first cancer in Tuscan women

\begin{tabular}{|c|c|c|c|c|c|c|c|c|c|c|c|c|c|}
\hline \multirow[b]{2}{*}{ First cancer } & \multicolumn{4}{|c|}{$\begin{array}{l}\text { Ipsilateral breast relapse } \\
\text { (\% from } 455)\end{array}$} & \multicolumn{4}{|c|}{$\begin{array}{c}\text { Contralateral breast cancer } \\
\text { (\% from } 589)\end{array}$} & \multicolumn{4}{|c|}{$\begin{array}{l}\text { Subjects with second cancer } \\
\text { (\% from 1044) }\end{array}$} & \multirow[b]{2}{*}{ Total (\%) } \\
\hline & pTis & pTIa-c & pT2+ & pTx & pTis & pTIa-c & pT2+ & pTx & pTis & pTla-c & pT2+ & pTx & \\
\hline pTla-c & 33 & 168 & 43 & 13 & 27 & 199 & 34 & 11 & 60 & 367 & 77 & 24 & $528(5 \mathrm{I})$ \\
\hline PT2+ & 5 & 52 & 17 & 11 & 15 & $|4|$ & 55 & 16 & 20 & 193 & 72 & 27 & $312(30)$ \\
\hline pTx & 0 & 19 & 4 & 5 & 7 & 36 & 6 & 5 & 7 & 55 & 10 & 10 & $82(8)$ \\
\hline
\end{tabular}

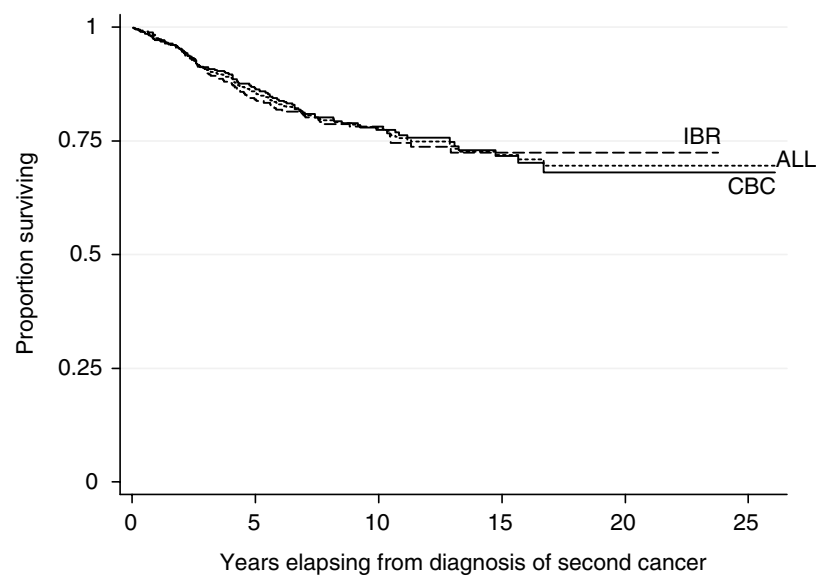

Figure I Kaplan-Meier disease-specific survival curves for ipsilateral breast relapse (IBR), contralateral breast cancer (CBC) and all second cancers.

recommended follow-up of 10 years or even longer may be more appropriate (Smith et al, 1999; Khatcheressian et al, 2006). A recent study from Edinburgh, based on 108 relapses (including 35 cases of $\mathrm{CBC}$ ) makes a similar point, and reports that treatable breast relapse occurred at a constant rate for at least 10 years (Montgomery et al, 2007). The benefits of extending surveillance must be weighed against potential disadvantages in terms of overdiagnosis and overtreatment although these effects are likely to be small for this cohort.

Surgical management of CBC was more often breast conservation than mastectomy, in keeping with the preponderance of small cancers in this group. In addition, most women with CBC had node-negative disease, although data on nodal status were not known in about a quarter of cases (nodes either not excised or data not notified to the study centre). Although local excision of in-breast relapse is associated with lower local control than salvage mastectomy (van der Sangen et al, 2006), a $40 \%$ breast conservation rate in women with IBR in our study might reflect feasibility of local excision as $62 \%$ were small tumours.

Our data do not represent complete population data; however, the study centre is the region's main breast diagnostic service with linkage to population registries, and is the only service in the Tuscan region which provides organised surveillance for women with a past $\mathrm{BC}$ history. We therefore consider that the majority of women in our case definition will have been identified and included. It is also relevant that our study concerned subjects with two BCs and does not allow calculation of predictors of the occurrence of a second cancer event nor rates of IBR and CBC. These were not within the scope of this evaluation; such data on these issues have been previously reported (Kurtz et al, 1988; Rack et al, 2003; Montgomery et al, 2007).
One of the clear findings of this study is that the majority of second BCs was detected in asymptomatic women. These data suggest that 'early detection' of the second cancer may be occurring during routine follow-up of women with BC. A recent case-control study found mammography surveillance was associated with improved survival in older BC survivors (Lash et al, 2007). As we are considering survival from diagnosis of the second cancer, estimation of the effect of early detection (based on symptom status) is likely to be subject to lead-time and length biases. For this reason we have not included this variable in survival analysis for purposes of this study. However, we are currently collecting additional data on symptoms and mode of diagnosis of second cancers in our cohort to validly quantify the extent and potential impact of early detection in women with second cancer events.

The high proportion of asymptomatic second cancers is consistent with finding that overall second cancers were smaller than first cancers, although clearly evident for only CBC. As CBC is essentially a new cancer event, screening may play a larger role in its diagnosis. Ipsilateral breast relapses are predominantly recurrences of the initial tumour and so the observed differences in tumour size may reflect the differing biological nature of these two cancer events.

Histological types of tumours, for both the first and second cancer, also differed in distribution between IBR and CBC. This needs to be interpreted in the context of the long timeframe of the study (with possible variability in pathology reporting criteria and pathologists) and that some types were not specified. The interesting aspect of differences in the distribution of tumour histology is that it was largely determined (in second cancers) by a significantly higher proportion of DCIS in IBR than CBC, and a significantly higher proportion of invasive lobular cancer in $\mathrm{CBC}$ than IBR. Differences in distribution of histology of the first cancer were similarly influenced by a significantly higher proportion of DCIS in IBR than CBC.

Poorer disease-specific survival was associated with shorter times from the first to second cancer event, diagnosis of the second cancer in an earlier time period, second cancer tumour size $\geqslant 2 \mathrm{~cm}$, and positive nodes. The association between poorer prognosis and short time from the initial cancer to in-breast relapse is well known, but such an association has not previously been clearly demonstrated for CBC (Ciatto et al, 2004). The association between earlier epoch of diagnosis and poorer survival has been reported by others in analyses of population data in both primary early and metastatic BC (Chen et al, 2007; Ernst et al, 2007; Dabakuyo et al, 2008), so we presume this is mainly indicative of therapy effect. It is also possible that some of this effect relates to early detection.

Although all the prognostic variables we have described are relevant to clinicians providing care to women with a second $\mathrm{BC}$, based on the strongest predictor variables for survival in our model, $\mathrm{pT}$ and $\mathrm{pN}$ categories are likely to be more clinically relevant in prognostication, although the occurrence 
Table 3 Disease-specific survival analysis following diagnosis of a second breast cancer: multivariate Cox proportional hazards model

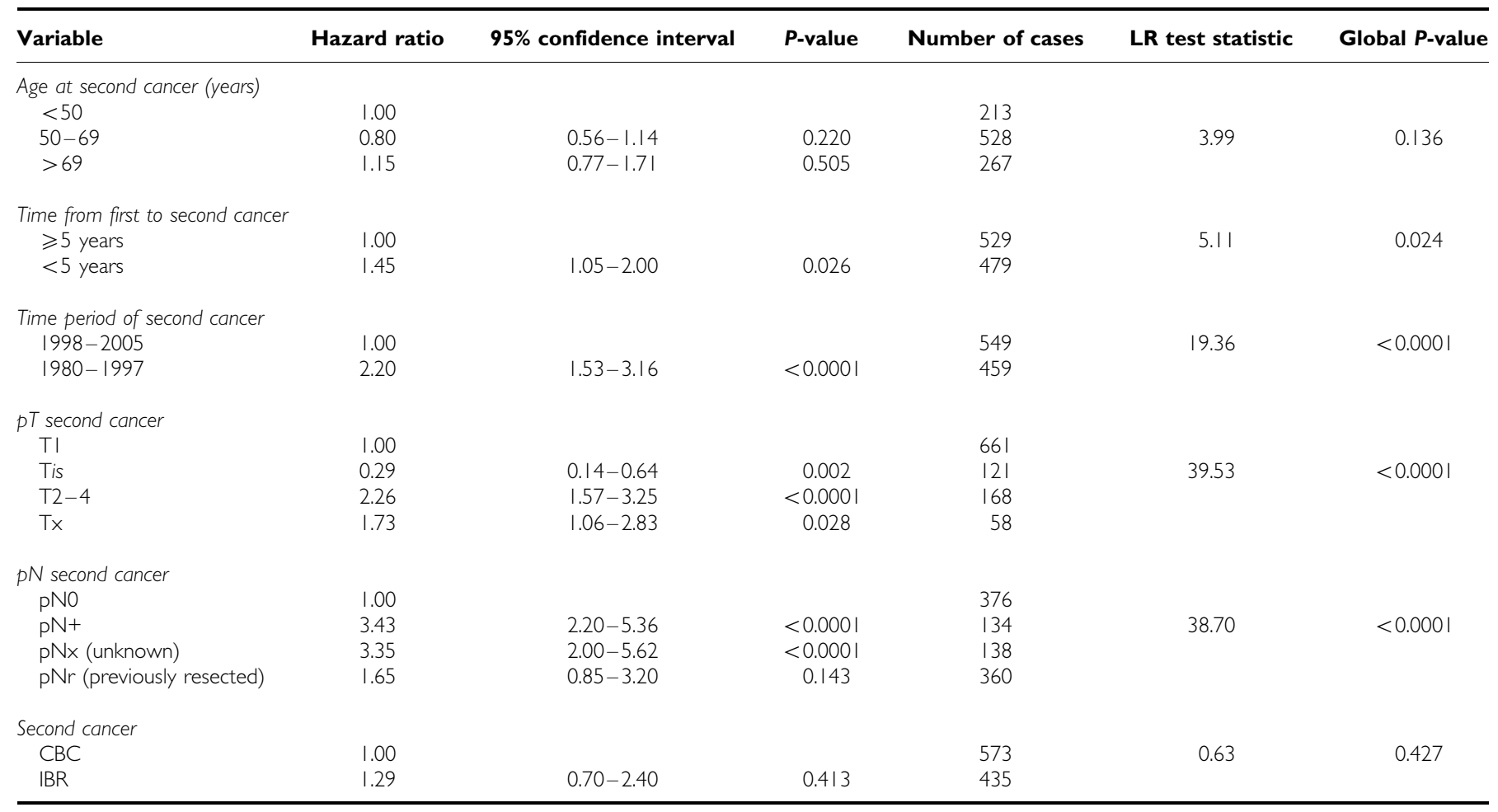

Table 4 Multivariate logistic regression for assessing features of the first breast cancer associated with large or node-positive second breast cancers

\begin{tabular}{|c|c|c|c|c|c|c|c|c|}
\hline \multirow[b]{2}{*}{ First cancer variable } & \multicolumn{4}{|c|}{ Predictive of second cancer with tumour size $\geqslant 2 \mathrm{~cm}$} & \multicolumn{4}{|c|}{ Predictive of second cancer with positive nodes } \\
\hline & $\begin{array}{l}\text { Odds ratio } \\
(95 \% \mathrm{Cl})\end{array}$ & $P$-value & $\begin{array}{l}\text { LR test } \\
\text { statistic }\end{array}$ & $\begin{array}{l}\text { Global } \\
\text { P-value }\end{array}$ & $\begin{array}{l}\text { Odds ratio } \\
(95 \% \mathrm{Cl})\end{array}$ & $P$-value & $\begin{array}{l}\text { LR test } \\
\text { statistic }\end{array}$ & $\begin{array}{l}\text { Global } \\
P \text {-value }\end{array}$ \\
\hline \multicolumn{9}{|l|}{ Age at first cancer (years) } \\
\hline$<50$ & 1.00 & & & & 1.00 & & & \\
\hline $50-69$ & $0.96(0.67-1.38)$ & 0.837 & 1.85 & 0.396 & $0.76(0.52-1.12)$ & 0.171 & 3.71 & 0.157 \\
\hline$>69$ & $1.37(0.82-2.28)$ & 0.234 & & & $0.57(0.29-1.11)$ & 0.097 & & \\
\hline \multicolumn{9}{|l|}{ pT of first cancer } \\
\hline $\mathrm{Ti}$ & 1.00 & & & & 1.00 & & & \\
\hline Tis & $1.67(0.44-6.38)$ & 0.452 & 7.46 & 0.059 & $2.18(0.57-8.35)$ & 0.254 & 17.36 & 0.0006 \\
\hline $\mathrm{T} 2-4$ & $1.54(1.06-2.23)$ & 0.023 & & & $2.44(1.59-3.75)$ & $<0.0001$ & & \\
\hline Tx & $0.75(0.37-1.55)$ & 0.439 & & & $1.40(0.68-2.90)$ & 0.360 & & \\
\hline \multicolumn{9}{|l|}{ pN of first cancer } \\
\hline pNO & 1.00 & & & & 1.00 & & & \\
\hline Invasive ductal & 1.00 & & & & 1.00 & & & \\
\hline DCIS & $0.44(0.11-1.73)$ & 0.241 & 2.27 & 0.518 & $0.63(0.16-2.44)$ & 0.506 & 4.35 & 0.227 \\
\hline Invasive, special types & $0.72(0.31-1.63)$ & 0.427 & & & $2.10(1.02-4.32)$ & 0.043 & & \\
\hline Invasive (other) ${ }^{\mathrm{a}}$ & $1.11(0.73-1.68)$ & 0.620 & & & $1.08(0.68-1.73)$ & 0.737 & & \\
\hline
\end{tabular}

${ }^{\mathrm{a} C}$ Cases where histological type of invasive breast malignancy was not specified and those with missing data on type of cancer histology.

of a second cancer soon after the first may be a warning sign of a more aggressive tumour and poorer outcome. The most powerful predictive features of the first $\mathrm{BC}$, in terms of predicting second cancers with poor prognostic features (second cancer tumour size $\geqslant 2 \mathrm{~cm}$ or positive nodes) were larger tumour size and positive nodes. A larger first $\mathrm{BC}(\geqslant 2 \mathrm{~cm})$ was a significant predictor of node metastases on diagnosis of the second cancer.

\section{ACKNOWLEDGEMENTS}

Dr Houssami is funded by National Health and Medical Research Council (NHMRC) program Grant no. 402764 to the Screening and Test Evaluation Program.

\section{Conflict of interest}

None. 


\section{REFERENCES}

Chen SL, Hoehne FM, Giuliano AE (2007) The prognostic significance of micrometastases in breast cancer: a SEER population-based analysis. Ann Surg Oncol 14: 3378 - 3384

Ciatto S, Miccinesi G, Zappa M (2004) Prognostic impact of the early detection of metachronous contralateral breast cancer. Eur J Cancer 40: $1496-1501$

Dabakuyo TS, Bonnetain F, Roignot P, Poillot ML, Chaplain G, Altwegg T, Hedelin G, Arveux P (2008) Population-based study of breast cancer survival in Cote d'Or (France): prognostic factors and relative survival. Ann Oncol 19: 276-283

Ernst MF, van de Poll-Franse LV, Roukema JA, Coebergh JW, van Gestel CM, Vreugdenhil G, Louwman MJ, Voogd AC (2007) Trends in the prognosis of patients with primary metastatic breast cancer diagnosed between 1975 and 2002. Breast 16: $344-351$

Grunfeld E, Noorani H, McGahan L, Paszat L, Coyle D, van Walraven C, Joyce J, Sawka C (2002) Surveillance mammography after treatment of primary breast cancer: a systematic review. Breast 11: 228-235

Khatcheressian JL, Wolff AC, Smith TJ, Grunfeld E, Muss HB, Vogel VG, Halberg F, Somerfield MR, Davidson NE (2006) American Society of Clinical Oncology 2006 update of the breast cancer follow-up and management guidelines in the adjuvant setting. J Clin Oncol 24: $5091-5097$

Kurtz JM, Amalric R, Brandone H, Ayme Y, Spitalier JM (1988) Contralateral breast cancer and other second malignancies in patients treated by breast-conserving therapy with radiation. Int J Radiat Oncol Biol Phys 15: $277-284$

Lash TL, Fox MP, Buist DS, Wei F, Field TS, Frost FJ, Geiger AM, Quinn VP, Yood MU, Silliman RA (2007) Mammography surveillance and mortality in older breast cancer survivors. J Clin Oncol 25: $3001-3006$
Montgomery DA, Krupa K, Jack WJ, Kerr GR, Kunkler IH, Thomas J, Dixon JM (2007) Changing pattern of the detection of locoregional relapse in breast cancer: the Edinburgh experience. Br J Cancer 96: 1802-1807

National Institute for Clinical Excellence (2002) Guidance on cancer services: Improving Outcomes in Breast Cancer -Manual update. www.nice.org.uk (accessed 22nd November 2007)

Parkin DM, Bray F, Ferlay J, Pisani P (2005) Global cancer statistics, 2002. CA Cancer J Clin 55: 74-108

Rack B, Janni W, Gerber B, Strobl B, Schindlbeck C, Klanner E, Rammel G, Sommer H, Dimpfl T, Friese K (2003) Patients with recurrent breast cancer: does the primary axillary lymph node status predict more aggressive tumor progression? Breast Cancer Res Treat 82: 83-92

Schootman M, Fuortes L, Aft R (2006) Prognosis of metachronous contralateral breast cancer according to stage at diagnosis: the importance of early detection. Breast Cancer Res Treat 99: 91-95

Smith TJ, Davidson NE, Schapira DV, Grunfeld E, Muss HB, Vogel III VG, Somerfield MR (1999) American Society of Clinical Oncology 1998 update of recommended breast cancer surveillance guidelines. J Clin Oncol 17: $1080-1082$

Tabar L, Fagerberg G, Duffy SW, Day NE, Gad A, Grontoft O (1992) Update of the Swedish two-county program of mammographic screening for breast cancer. Radiol Clin N Amer 30(1): 187-210

The Association of Breast Surgery @ BASO, Royal College of Surgeons of England (2005) Guidelines for the management of symptomatic breast disease. Eur J Surg Oncol 31(Suppl 1): 1-21

van der Sangen MJ, van de Poll-Franse LV, Roumen RM, Rutten HJ, Coebergh JW, Vreugdenhil G, Voogd AC (2006) The prognosis of patients with local recurrence more than five years after breast conservation therapy for invasive breast carcinoma. Eur J Surg Oncol 32: $34-38$ 